\title{
Convergence Analysis of Wireless Remote Iterative Learning Control Systems with Dropout Compensation
}

\author{
Li-xun Huang ${ }^{1}$ and Yong Fang ${ }^{1,2}$ \\ ${ }^{1}$ School of Communication and Information Engineering, Key Laboratory of Specialty Fiber Optics and Optical Access Networks, \\ Shanghai University, Shanghai 200072, China \\ ${ }^{2}$ Key Laboratory of Advanced Display and System Applications, Shanghai University, Shanghai 200072, China
}

Correspondence should be addressed to Li-xun Huang; shuhlx@gmail.com

Received 12 December 2012; Revised 5 March 2013; Accepted 6 March 2013

Academic Editor: Mohammad Younis

Copyright ( 2013 L.-x. Huang and Y. Fang. This is an open access article distributed under the Creative Commons Attribution License, which permits unrestricted use, distribution, and reproduction in any medium, provided the original work is properly cited.

\begin{abstract}
The wireless remote iterative learning control (ILC) system with random data dropouts is considered. The data dropout is viewed as a binary switching sequence which obeys the Bernoulli distribution. In order to eliminate the effect of data dropouts on the convergence property of output error, the signal at the same time with the lost one but in the last iteration is used to compensate the data dropout at the actuator. With the dropout compensation, the convergence property of output error is analyzed by studying the element values of system transition matrix. Finally, some simulation results are given to illustrate the validity of the proposed method.
\end{abstract}

\section{Introduction}

One feature of the wireless remote control system is that signals are transmitted in wireless network from the sensor to the controller and from the controller to the actuator, and then the controller is separated from the system platform [1-3]. Consequently, the system has such advantages as easy installation, reduced wiring, and maintenance. In wireless remote control systems, designing a remote controller to track the desired trajectory is not an easy task. Fortunately, when systems execute the same task periodically in a fixed time interval, iterative learning control (ILC) is an effective method [4]. This method uses information obtained from the previous operation to improve the control signal for the next trial. If some conditions are met, the output error could monotonically converge to zero from iteration to iteration.

However, the introduction of wireless network makes the tracking of desired trajectory more complicated than traditional point-to-point connection method due to the unreliability of wireless network. The main issue is that some signals would be lost during transmitting, which can be divided into two different types: control signal dropouts and measurement signal dropouts. The first one occurs when the signal is transmitted from the controller to the actuator after updating, which would disturb the learning process indirectly; the second one arises when the signal is transmitted from the sensor to the controller, which would be involved in the learning process directly. The convergence of output error cannot be guaranteed without considering data dropouts.

The research on networked control systems has attracted much attention. In [5-9], authors are concerned with the state estimation problem for networked control systems with various uncertainties of the communication channels. In $[10,11]$, the author discussed decentralized stabilization of networked control systems with nonlinear perturbations. In [12], the nonfragile state feedback $H \infty$ control problem for networked control systems with quantized signals is studied. However, most researches related to the case with data dropouts are conducted only in sensor-to-controller side and did not consider the control scheme adopted by controller. In the very recent years, some researches on ILC systems considering the 
problem of data dropout appeared (see [13-21] and references therein). Liu et al. investigated the implementation of ILC in a remote control systems environment and specifically focused on compensation when both random data dropouts and delays occur at the communication network between the plant output and the controller [13]. In [14], the author proposed an averaging ILC algorithm to overcome the random transport delay and data dropout, which guarantees the convergence property of the ensemble average of the output tracking errors along the iteration axis. In [15], $\mathrm{Bu}$ and Hou discussed the stability of ILC with data dropouts via asynchronous system and offered the stability condition in the form of linear matrix inequalities. Bu et al. also studied the stability of first and high order ILC with data dropout when the plant is subject to measurement signal dropouts [16]. Ahn et al. presented a mathematical formulation of the problem of robust ILC design when the system is subject to measurement signal dropouts and used the Kalman filtering approach to design a learning gain such that the system eventually converges to a desired trajectory if there are not complete data dropouts [17], but the results were restricted to the case when the network from sensor to controller has dependency. Aiming at this problem, the author considered discrete-time intermittent iterative learning controller with independent data dropouts in his further study [18]. However, all the previously mentioned researches only considered the dropout of measurement signals. In [19], the author considered the problem of ILC for a class of nonlinear systems with control signal dropouts and measurement signal dropouts, but the convergence analysis needs controller and actuator to know the received signal whether lost or not. In [20], a sampled-data ILC approach was proposed for a class of nonlinear networked control systems to deal with the existence of time delays and packet losses in control signal and measurement signal transmissions. In [21], Pan et al. assumed that the controller and the actuator are all event driven and analyzed the effect of packet loss from controller to actuator side and from sensor to controller side on the convergence property of output error. But the convergence is asymptotic because the control error at the actuator cannot converge to zero. To the best of our knowledge, no one has studied the compensation for the data dropouts in both control signals and measurement signals to guarantee the convergence property of output error. This observation is the motivation of the present paper.

As depicted in the second paragraph, there are two different kinds of data dropouts in wireless remote ILC systems. For the sake of convenience, we only consider the control signal dropouts in this paper, but the results can be extended to the measurement signal dropouts. The data dropout would be described as a binary sequence which obeys a Bernoulli distribution taking the value of one and zero with certain probability. In order to eliminate the effect of data dropouts on the convergence property of output error, we consider using the signal at the same time with the lost one but in the last iteration to compensate the data dropout at the actuator. The convergence performance of the output error with dropout compensation would be analyzed by studying eigenvalues and other elements in the lower triangular of the system transition matrix.

\section{Problem Formulations}

The discrete-time linear system is considered by the following equation:

$$
\begin{gathered}
\mathbf{x}_{k}(t+1)=\mathbf{A x}_{k}(t)+\mathbf{B} \mathbf{u}_{k}(t), \\
\mathbf{y}_{k}(t)=\mathbf{C x}_{k}(t),
\end{gathered}
$$

where $\mathbf{x}_{k}(t), \mathbf{u}_{k}(t)$, and $\mathbf{y}_{k}(t)$ are state, control, and output variables, respectively, and $\mathbf{A}, \mathbf{B}$, and $\mathbf{C}$ are the system matrices. The subscript $k=0,1,2, \ldots$ is used to denote iteration and $t \in[0, T]$ is used to denote time. The system operates repeatedly in the iteration domain with the desired trajectory $\mathbf{y}_{d}(t)$, which can be of the form

$$
\begin{gathered}
\mathbf{x}_{d}(t+1)=\mathbf{A} \mathbf{x}_{d}(t)+\mathbf{B} \mathbf{u}_{d}(t), \\
\mathbf{y}_{d}(t)=\mathbf{C} \mathbf{x}_{d}(t),
\end{gathered}
$$

where $\mathbf{u}_{d}(t)$ and $\mathbf{x}_{d}(t)$ are desired control and state. In order to track $\mathbf{y}_{d}(t)$ precisely, adopting ILC method at the controller is a useful method and the first order ILC can be expressed as

$$
\mathbf{u}_{k+1}(t)=\mathbf{u}_{k}(t)+\Gamma(t) \mathbf{e}_{k}(t+1),
$$

where $\boldsymbol{\Gamma}(t)$ is learning gain, and output error $\mathbf{e}_{k}(t)=\mathbf{y}_{d}(t)-$ $\mathbf{y}_{k}(t), t \in[0, T-1]$. The setup of wireless remote ILC systems is illustrated as in Figure $1[22,23]$. During the measurement signal $\mathbf{e}_{k}(t)$ transmitting from the sensor to the controller and the control signal $\mathbf{u}_{k}(t)$ transmitting from the controller to the actuator, data dropouts occur due to the unreliability of wireless network. Taking the effect of data dropouts into account, the system controlled remotely by iterative learning controller can be represented as

$$
\begin{gathered}
\mathbf{x}_{k}(t+1)=\mathbf{A} \mathbf{x}_{k}(t)+\mathbf{B} \widetilde{\mathbf{u}}_{k}(t), \\
\mathbf{y}_{k}(t)=\mathbf{C} \mathbf{x}_{k}(t), \\
\mathbf{u}_{k+1}(t)=\mathbf{u}_{k}(t)+\boldsymbol{\Gamma}(t) \widetilde{\mathbf{e}}_{k}(t+1),
\end{gathered}
$$

where control signal $\widetilde{\mathbf{u}}_{k}(t)$ received at the actuator and measurement signal $\widetilde{\mathbf{e}}_{k}(t+1)$ received at the controller can be expressed as

$$
\begin{aligned}
\widetilde{\mathbf{u}}_{k}(t) & =\xi_{k}(t) \mathbf{u}_{k}(t), \\
\widetilde{\mathbf{e}}_{k}(t+1) & =\eta_{k}(t) \mathbf{e}_{k}(t+1),
\end{aligned}
$$

where $\mathbf{u}_{k}(t)$ and $\mathbf{e}_{k}(t+1)$ are signals at the controller and the sensor, respectively. The stochastic parameters $\xi_{k}(t)$ and $\eta_{k}(t)$ are two scalar Bernoulli distributed random variables taking value 0 or 1 (i.e., $\left.\xi_{k}(t), \eta_{k}(t) \in\{0,1\}, \forall k, t\right)$. $\xi_{k}(t)$ is uncorrelated with $\eta_{k}(t)$. In this model, if the variable takes value 0 , then the data is lost correspondingly; otherwise there is no data dropout. 


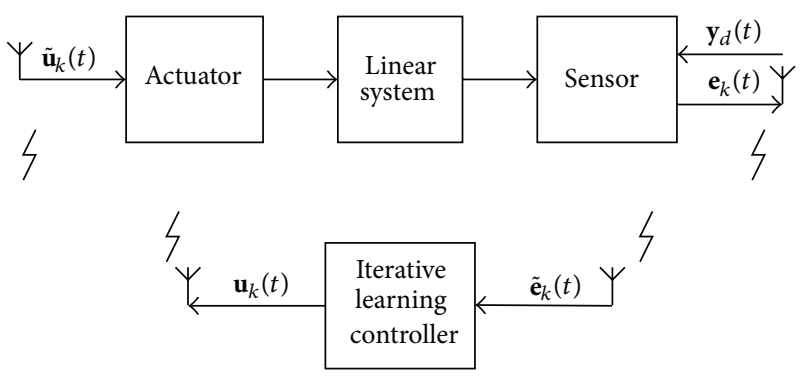

FIGURE 1: Block diagram of wireless remote ILC systems.

Random data dropouts could disturb the learning process of controller and have an effect on the convergence performance of output error. In order to remove the effect of data dropouts, a compensation method would be proposed in Section 3.

\section{The Data Dropout Compensation Scheme}

In this section, we will derive the relation between output error and control error at the actuator and the relation of control errors at the controller in different iterations first, and then we will propose a compensation scheme based on the relations and attribute of control error. In order to simplify the analysis, the following assumptions are made

Assumption 1. Consider $\|\boldsymbol{\Gamma}(t)\| \leq \beta_{\Gamma},\|\mathbf{A}\| \leq \beta_{A},\|\mathbf{B}\| \leq$ $\beta_{B},\|\mathbf{C}\| \leq \beta_{C}$.

Assumption 2. Consider $\mathbf{x}_{k}(0)=\mathbf{x}_{d}(0)$, for all $k$.

The output error $\mathbf{e}_{k}(t+1)$ can be represented as

$$
\begin{aligned}
\mathbf{e}_{k}(t+1) & =\mathbf{y}_{d}(t+1)-\mathbf{y}_{k}(t+1) \\
& =\mathbf{C} \delta \mathbf{x}_{k}(t+1)
\end{aligned}
$$

From (2) and (4), it is easy to find the nonrecursive solution to $\delta \mathbf{x}_{k}(t+1)$ as

$$
\begin{aligned}
\delta \mathbf{x}_{k}(t+1) & =\mathbf{A} \delta \mathbf{x}_{k}(t)+\mathbf{B} \delta \widetilde{\mathbf{u}}_{k}(t) \\
& =\mathbf{A}^{t+1} \delta \mathbf{x}_{k}(0)+\sum_{i=0}^{t} \mathbf{A}^{t-i} \mathbf{B} \delta \widetilde{\mathbf{u}}_{k}(i),
\end{aligned}
$$

where $\delta \widetilde{\mathbf{u}}_{k}(i)$ denotes the control error at the actuator. If Assumption 2 is satisfied, substitute (8) into (7) to get

$$
\mathbf{e}_{k}(t+1)=\sum_{i=0}^{t} \mathbf{C A}^{t-i} \mathbf{B} \delta \widetilde{\mathbf{u}}_{k}(i)
$$

Take norms on both sides of (9) to yield

$$
\left\|\mathbf{e}_{k}(t+1)\right\|=\sum_{i=0}^{t} \beta_{C} \beta_{A}^{t-i} \beta_{B}\left\|\delta \widetilde{\mathbf{u}}_{k}(i)\right\| .
$$

From (10), the relation between output error $\mathbf{e}_{k}(i), i \in$ $[1, T]$, and control error $\delta \widetilde{\mathbf{u}}_{k}(i), i \in[0, T-1]$, at the actuator can be expressed in the matrix form

$$
\begin{aligned}
{\left[\begin{array}{c}
\left\|\mathbf{e}_{k}(1)\right\| \\
\vdots \\
\left\|\mathbf{e}_{k}(T)\right\|
\end{array}\right]=} & {\left[\begin{array}{ccc}
\rho^{\prime}(0) & \cdots & 0 \\
\vdots & \ddots & \vdots \\
\rho^{\prime}(T-1) & \cdots & \rho^{\prime}(0)
\end{array}\right] } \\
& \times\left[\begin{array}{c}
\left\|\delta \widetilde{\mathbf{u}}_{k}(0)\right\| \\
\vdots \\
\left\|\delta \widetilde{\mathbf{u}}_{k}(T-1)\right\|
\end{array}\right]
\end{aligned}
$$

where $\rho^{\prime}(i)=\beta_{C} \beta_{A}^{i} \beta_{B}, i \in[0, T-1]$.

On the other hand, the relation of control errors at the controller in different iterations can be get from (5) as follows:

$$
\begin{aligned}
\delta \mathbf{u}_{k+1}(t) & =\mathbf{u}_{d}(t)-\mathbf{u}_{k+1}(t) \\
& =\mathbf{u}_{d}(t)-\mathbf{u}_{k}(t)-\Gamma(t) \widetilde{\mathbf{e}}_{k}(t+1) \\
& =\delta \mathbf{u}_{k}(t)-\Gamma(t) \widetilde{\mathbf{e}}_{k}(t+1) .
\end{aligned}
$$

If there are no signals happen data dropouts, $\widetilde{\mathbf{e}}_{k}(t+1)=$ $\mathbf{e}_{k}(t+1)$ and $\widetilde{\mathbf{u}}_{k}(t)=\mathbf{u}_{k}(t)$. Substituting (9) into (12), we have

$$
\delta \mathbf{u}_{k+1}(t)=\delta \mathbf{u}_{k}(t)-\boldsymbol{\Gamma}(t) \sum_{i=0}^{t} \mathbf{C A} A^{t-i} \mathbf{B} \delta \mathbf{u}_{k}(i) .
$$

Taking norms of both sides of (13), the relation can be rewritten as

$$
\begin{array}{r}
\left\|\delta \mathbf{u}_{k+1}(t)\right\| \leq \mathbf{H}_{k+1, k}(t) \mathbf{H}_{k, k-1}(t) \psi_{k-1}(t), \\
t \in[0, T-1],
\end{array}
$$

where the vector of control error $\psi_{k-1}, \mathbf{H}_{k+1, k}(t)$, and transition matrix $\mathbf{H}_{k, k-1}(t)$ can be represented as (15), (16), and (17), respectively

$$
\begin{aligned}
& \psi_{k-1}(t)=\left[\left\|\delta \mathbf{u}_{k-1}(0)\right\|,\left\|\delta \mathbf{u}_{k-1}(1)\right\|, \ldots,\left\|\delta \mathbf{u}_{k-1}(t)\right\|\right]^{T} \\
& \mathbf{H}_{k+1, k}(t)=\left[\begin{array}{llll}
\rho_{1}(t) & \cdots & \rho_{1}(1) & \rho_{0}(t)
\end{array}\right], \\
& \mathbf{H}_{k, k-1}(t)=\left[\begin{array}{cccc}
\rho_{0}(0) & 0 & \cdots & 0 \\
\rho_{1}(1) & \rho_{0}(1) & \ddots & \vdots \\
\vdots & \ddots & \ddots & 0 \\
\rho_{1}(t) & \cdots & \rho_{1}(1) & \rho_{0}(t)
\end{array}\right] \text {, }
\end{aligned}
$$

where $\rho_{1}(i)=\beta_{\Gamma} \beta_{C} \beta_{A}^{i} \beta_{B}$ and $\rho_{0}(i)=\|\mathbf{I}-\boldsymbol{\Gamma}(i) \mathbf{C B}\|, i \in[0, t]$. 
If $\widetilde{\mathbf{e}}_{k}(t+1)=\mathbf{e}_{k}(t+1)$ and $\widetilde{\mathbf{u}}_{k}(t)=\mathbf{u}_{k}(t)$ are satisfied, the convergence of $\left\|\mathbf{e}_{k}(t+1)\right\|$ is determined by $\left\|\delta \mathbf{u}_{k}(i)\right\|, i \in$ $[0, t]$, and the convergence of $\left\|\delta \mathbf{u}_{k}(t)\right\|$ is determined by the eigenvalues of transition matrix. From (14), we have $\left\|\delta \mathbf{u}_{k+1}(t)\right\| \leq \mathbf{H}_{k+1, k}(t) \prod_{i=0}^{k} \mathbf{H}_{i+1, i}(t) \psi_{0}(t)$. Since $\mathbf{H}_{i+1, i}(t)$ is a lower triangular matrix, all of its eigenvalues are the diagonal elements $\rho_{0}(i)$. Selecting the learning gain $\Gamma(t)$ such that all eigenvalues of $\mathbf{H}_{i+1, i}(t)$ are within unit circle; that is, $\rho_{0}(i)=\|\mathbf{I}-\boldsymbol{\Gamma}(i) \mathbf{C B}\|<1, \lim _{k \rightarrow \infty}\left\|\delta \mathbf{u}_{k+1}(t)\right\|=0, t \in$ $[0, T-1]$, and then $\lim _{k \rightarrow \infty}\left\|\mathbf{e}_{k}(t)\right\|=0$, for all $t \in[1, T]$, is guaranteed. However, with the introduction of random data dropouts in control signals, $\widetilde{\mathbf{u}}_{k}(t) \neq \mathbf{u}_{k}(t)$, the element values of $\mathbf{H}_{k+1, k}(t)$ and $\mathbf{H}_{i+1, i}(t)$ would be changed, so the convergence of $\left\|\delta \mathbf{u}_{k}(t)\right\|$ cannot be guaranteed and then they have an effect on the convergence performance of output error $\left\|\mathbf{e}_{k}(t+1)\right\|$.

In order to remove the effect, we propose to use the control signal at same time with the lost one but in the last iteration to compensate the data dropout at the actuator based on the attribute that control signal converges in the iteration domain, which can be represented by

$$
\widetilde{\mathbf{u}}_{k}(t)=\xi_{k}(t) \mathbf{u}_{k}(t)+\left(1-\xi_{k}(t)\right) \mathbf{u}_{k-1}(t) .
$$

The convergence of output error with the proposed compensation scheme would be analyzed in the next section.

\section{Convergence Analysis of Output Error with Data Dropout Compensation}

In the following part, we start by assuming that there is one control signal $\mathbf{u}_{k}(t)$ loss during the $k$ th iteration and that $\mathbf{u}_{k-1}(t)$ is applied to compensate the lost $\mathbf{u}_{k}(t)$ at the actuator, and then we extend it to the condition with multiple control signals loss. The convergence property of output error with data dropout compensation can be concluded in the following theorem.

Theorem 1. With the compensation method presented in (18), if the condition $0<\rho(\boldsymbol{\Gamma}(t) \mathbf{C B})<1$ is satisfied, then

$$
\lim _{k \rightarrow \infty}\left\|\mathbf{e}_{k}(t)\right\|=0, \quad \forall t \in[1, T] .
$$

Proof. If the control signal $\mathbf{u}_{k}(t)$ is lost, $\mathbf{u}_{k-1}(t)$ would be applied at the actuator to compensate the dropout of $\mathbf{u}_{k}(t)$. In this condition, output errors $\mathbf{e}_{k}(i),(0 \leq i \leq t)$ are not affected but $\mathbf{e}_{k}(i),(t+1 \leq i \leq T)$ are all changed according to (11). Consequently, the proof can be divided into the following two parts due to changing $\mathbf{e}_{k}(t+1), \mathbf{e}_{k}(t+2)$ and afterwards.

(1) Convergence Analysis of $\left\|\mathbf{e}_{k}(t+1)\right\|$ with Dropout Compensation.

According to $\mathbf{e}_{k}(t)=\mathbf{y}_{d}(t)-\mathbf{y}_{k}(t)$, the output error $\mathbf{e}_{k}(t+1)$ can be represented as

$$
\begin{aligned}
\mathbf{e}_{k}(t+1) & =\mathbf{y}_{d}(t+1)-\mathbf{y}_{k}(t+1) \\
& =\mathbf{C} \delta \mathbf{x}_{k}(t+1) .
\end{aligned}
$$

Using (2), (4), and Assumption 2, the relation between state error $\delta \mathbf{x}_{k}(t)$ and control error $\delta \mathbf{u}_{k}(t)$ can be expressed as

$$
\begin{aligned}
\delta \mathbf{x}_{k}(t+1) \\
=\mathbf{x}_{d}(t+1)-\mathbf{x}_{k}(t+1) \\
=\mathbf{A} \delta \mathbf{x}_{k}(t)+\mathbf{B}\left(\xi_{k}(t) \delta \mathbf{u}_{k}(t)+\left(1-\xi_{k}(t)\right) \delta \mathbf{u}_{k-1}(t)\right) \\
=\sum_{i=0}^{t-1} \mathbf{A}^{t-i} \mathbf{B}\left(\xi_{k}(i) \delta \mathbf{u}_{k}(i)+\left(1-\xi_{k}(i)\right) \delta \mathbf{u}_{k-1}(i)\right) .
\end{aligned}
$$

From (5), (20), (21), and $\widetilde{\mathbf{e}}_{k}(t+1)=\mathbf{e}_{k}(t+1)$, the relation of control errors in different iterations can be represented as

$$
\begin{aligned}
\delta \mathbf{u}_{k+1} & (t) \\
= & \mathbf{u}_{d}(t)-\mathbf{u}_{k+1}(t) \\
= & \mathbf{u}_{d}(t)-\mathbf{u}_{k}(t)-\boldsymbol{\Gamma}(t) \widetilde{\mathbf{e}}_{k}(t+1) \\
= & \delta \mathbf{u}_{k}(t)-\boldsymbol{\Gamma}(t) \mathbf{C} \delta \mathbf{x}_{k}(t+1) \\
= & \delta \mathbf{u}_{k}(t)-\boldsymbol{\Gamma}(t) \\
& \times \sum_{i=0}^{t} \mathbf{C A}^{t-i} \mathbf{B}\left(\xi_{k}(i) \delta \mathbf{u}_{k}(i)+\left(1-\xi_{k}(i)\right) \delta \mathbf{u}_{k-1}(i)\right) .
\end{aligned}
$$

If the control signal $\mathbf{u}_{k}(t)$ is lost, the random parameter $\xi_{k}(t)=0$ correspondingly, then (22) could be changed to

$$
\begin{aligned}
\delta \mathbf{u}_{k+1}(t)= & \delta \mathbf{u}_{k}(t)-\boldsymbol{\Gamma}(t) \mathbf{C B} \delta \mathbf{u}_{k-1}(t) \\
& -\boldsymbol{\Gamma}(t) \sum_{i=0}^{t-1} \mathbf{C} \mathbf{A}^{t-i} \mathbf{B} \delta \mathbf{u}_{k}(i) .
\end{aligned}
$$

We can show $\delta \mathbf{u}_{k+1}(t)$ using control error in $(k-1)$ st iteration further, which is given by

$$
\begin{aligned}
\delta \mathbf{u}_{k+1}(t) & \\
= & (\mathbf{I}-2 \boldsymbol{\Gamma}(t) \mathbf{C B}) \delta \mathbf{u}_{k-1}(t) \\
& -\boldsymbol{\Gamma}(t) \sum_{i=0}^{t-1} \mathbf{C} \mathbf{A}^{t-i} \mathbf{B} \delta \mathbf{u}_{k-1}(i) \\
& -\boldsymbol{\Gamma}(t) \sum_{i=0}^{t-1} \mathbf{C A}{ }^{t-i} \mathbf{B}\left(\delta \mathbf{u}_{k-1}(i)-\boldsymbol{\Gamma}(i) \sum_{j=0}^{i} \mathbf{C A}^{i-j} \mathbf{B} \delta \mathbf{u}_{k-1}(j)\right) .
\end{aligned}
$$

Furthermore, (24) could be rewritten in the form of transition matrix 


$$
\delta \mathbf{u}_{k+1}(t)=\left[\begin{array}{c}
\boldsymbol{\Gamma}(t) \mathbf{C} \mathbf{A}^{t} \mathbf{B} \\
\vdots \\
\vdots \\
\boldsymbol{\Gamma}(t) \mathbf{C A B} \\
\mathbf{I}
\end{array}\right]^{T}\left[\begin{array}{ccccc}
\mathbf{I}-\boldsymbol{\Gamma}(0) \mathbf{C B} & 0 & \cdots & \cdots & 0 \\
\Gamma(1) \mathbf{C A B} & \ddots & \ddots & & \vdots \\
\vdots & \vdots & \ddots & \ddots & \vdots \\
\Gamma(t-1) \mathbf{C A}^{t-1} \mathbf{B} & \cdots & \boldsymbol{\Gamma}(t-1) \mathbf{C A B} & \mathbf{I}-\boldsymbol{\Gamma}(t-1) \mathbf{C B} & 0 \\
\Gamma(t) \mathbf{C A}^{t} \mathbf{B} & \cdots & \cdots & \boldsymbol{\Gamma}(t) \mathbf{C A B} & \mathbf{I}-2 \boldsymbol{\Gamma}(t) \mathbf{C B}
\end{array}\right]\left[\begin{array}{c}
\delta \mathbf{u}_{k-1}(0) \\
\vdots \\
\vdots \\
\vdots \\
\delta \mathbf{u}_{k-1}(t)
\end{array}\right] .
$$

Taking norms of both sides of (25), we have

$$
\begin{gathered}
\left\|\delta \mathbf{u}_{k+1}(t)\right\| \leq \mathbf{H}_{k+1, k}^{\prime}(t) \mathbf{H}_{k, k-1}^{\prime}(t) \psi_{k-1}(t), \\
\mathbf{H}_{k, k-1, k}^{\prime}(t)=\left[\begin{array}{lllll}
\rho_{1}(t) & \cdots & \rho_{1}(1) & 1
\end{array}\right], \\
{\left[\begin{array}{ccccc}
\rho_{0}(0) & 0 & \cdots & \cdots & 0 \\
\rho_{1}(1) & \ddots & \ddots & & \vdots \\
\vdots & \vdots & \ddots & \ddots & \vdots \\
\rho_{1}(t-1) & \cdots & \rho_{1}(1) & \rho_{0}(t-1) & 0 \\
\rho_{1}(t) & \cdots & \cdots & \rho_{1}(1) & \rho_{2}
\end{array}\right],}
\end{gathered}
$$

where $\rho_{2}=\|\mathbf{I}-2 \boldsymbol{\Gamma}(t) \mathbf{C B}\|$.

Clearly, $\mathbf{H}_{k+1, k}(t)$ in (16) becomes $\mathbf{H}_{k+1, k}^{\prime}(t)$ in which the element in $(t+1)$ st column is changed from $\rho_{0}(t)$ to 1 , and what is more is that transition matrix $\mathbf{H}_{k, k-1}^{\prime}(t)$ in (17) becomes $\mathbf{H}_{k, k-1}^{\prime}(t)$ in which the eigenvalue in $(t+1)$ st row is changed from $\rho_{0}(t)$ to $\rho_{2}$. If learning gain $\Gamma(t)$ is selected to satisfy the condition $0<\rho(\boldsymbol{\Gamma}(t) \mathbf{C B})<1$, then $0<1-$ $\rho(\boldsymbol{\Gamma}(t) \mathbf{C B})<1$ and $-1<1-2 \rho(\boldsymbol{\Gamma}(t) \mathbf{C B})<0$ are also satisfied, which indicate that $\|\mathbf{I}-\boldsymbol{\Gamma}(t) \mathbf{C B}\|<1$ and $\|\mathbf{I}-2 \boldsymbol{\Gamma}(t) \mathbf{C B}\|<1$. Since $\mathbf{H}_{k, k-1}^{\prime}(t)$ is a lower triangular matrix and all of its eigenvalues are the diagonal elements $\rho_{0}(i), 0 \leq i \leq t-1$ and $\rho_{2}$, we have $\lambda_{m}\left[\left(\prod_{i=0}^{k-2} \mathbf{H}_{i+1, i}(t)\right) \mathbf{H}_{k, k-1}^{\prime}(t)\left(\prod_{i=k}^{\infty} \mathbf{H}_{i+1, i}(t)\right)\right] \rightarrow 0, m=$ $0, \ldots, t+1$, which guarantees that $\lim _{k \rightarrow \infty}\left\|\delta \mathbf{u}_{k+1}(t)\right\|=0$.

According to the previous observation, control signals lost at time $t$ in each iteration will result in more " $\rho_{2}$ " in the diagonal elements of transition matrix $\mathbf{H}_{i+1, i}^{\prime}(t), 0 \leq i \leq$ $\infty$. As the structure of $\mathbf{H}_{i+1, i}^{\prime}(t)$ remains the lower triangular form, the structure of the serial product of transition matrix $\mathbf{H}_{i+1, i}^{\prime}(t)$ stays the lower triangular form as well. As a result, we can conclude that $\lambda_{m}\left[\prod_{i=0}^{\infty} \mathbf{H}_{i+1, i}^{\prime}(t)\right] \rightarrow 0, m=0,1, \ldots t$, which indicates $\lim _{k \rightarrow \infty}\left\|\delta \mathbf{u}_{k+1}(t)\right\|=0$.

Afterwards, we should highlight that if $\mathbf{u}_{k}(t)$ happens data dropout and $\mathbf{u}_{k-1}(t)$ is used the compensate the lost $\mathbf{u}_{k}(t)$, the control signal $\widetilde{\mathbf{u}}_{k}(t)$ at the actuator could also converge as the convergence of control signal $\mathbf{u}_{k}(t)$; that is, $\lim _{k \rightarrow \infty}\left\|\delta \widetilde{\mathbf{u}}_{k}(t)\right\|=\lim _{k \rightarrow \infty}\left\|\mathbf{u}_{d}(t)-\mathbf{u}_{k-1}(t)\right\|=0$ because $\lim _{k \rightarrow \infty} \mathbf{u}_{k}(t)=\lim _{k \rightarrow \infty} \mathbf{u}_{k-1}(t)$. In other words, the control signal not only at the controller but also at the actuator converge as iteration goes on. Furthermore, we know that the output error $\left\|\mathbf{e}_{k}(t+1)\right\|$ is a function of control errors $\left\|\delta \widetilde{\mathbf{u}}_{k}(i)\right\|$, $i \in[0, t]$ from (11), so the convergence of $\left\|\delta \widetilde{\mathbf{u}}_{k}(t)\right\|$ indicates $\lim _{k \rightarrow \infty}\left\|\mathbf{e}_{k}(t+1)\right\|=0$.

(2) Convergence Analysis of $\left\|\mathbf{e}_{k}(t+1+i)\right\|, 1 \leq i \leq T-t-1$ with Dropout Compensation.

In this part, we continue to analyze the convergence of $\| \mathbf{e}_{k}(t+$ $1+i) \|, 1 \leq i \leq T-t-1$. Due to the similarity in the analysis process, we would prove the convergence of $\left\|\mathbf{e}_{k}(t+2)\right\|$ as the previous part and prove the convergence of $\left\|\mathbf{e}_{k}(t+1+i)\right\|$, $2 \leq i \leq T-t-1$ by analogy.

Similarly, the output error $\mathbf{e}_{k}(t+2)$ can be represented as

$$
\begin{aligned}
\mathbf{e}_{k}(t+2) \\
=\mathbf{y}_{d}(t+2)-\mathbf{y}_{k}(t+2) \\
=\mathbf{C} \delta \mathbf{x}_{k}(t+2) \\
=\mathbf{C} \sum_{i=0}^{t+1} \mathbf{A}^{t+1-i} \mathbf{B}\left(\xi_{k}(i) \delta \mathbf{u}_{k}(i)+\left(1-\xi_{k}(i)\right) \delta \mathbf{u}_{k-1}(i)\right) .
\end{aligned}
$$

According to (5), (27), and $\widetilde{\mathbf{e}}_{k}(t+2)=\mathbf{e}_{k}(t+2), \delta \mathbf{u}_{k+1}(t+1)$ can be expressed as

$$
\begin{aligned}
\delta \mathbf{u}_{k+1} & (t+1) \\
= & \mathbf{u}_{d}(t+1)-\mathbf{u}_{k+1}(t+1) \\
= & \mathbf{u}_{d}(t+1)-\mathbf{u}_{k}(t+1)-\Gamma(t+1) \widetilde{\mathbf{e}}_{k}(t+2) \\
= & \delta \mathbf{u}_{k}(t)-\Gamma(t+1) \mathbf{C} \delta \mathbf{x}_{k}(t+2) \\
= & \delta \mathbf{u}_{k}(t+1)-\boldsymbol{\Gamma}(t+1) \\
& \times \sum_{i=0}^{t+1} \mathbf{C A}^{t+1-i} \mathbf{B}\left(\xi_{k}(i) \delta \mathbf{u}_{k}(i)+\left(1-\xi_{k}(i)\right) \delta \mathbf{u}_{k-1}(i)\right) .
\end{aligned}
$$

If the control signal $\mathbf{u}_{k}(t)$ is lost, $\xi_{k}(t)=0$ correspondingly, 
then (28) can be rewritten as

$$
\begin{aligned}
\delta \mathbf{u}_{k+1}( & t+1) \\
= & (\mathbf{I}-\boldsymbol{\Gamma}(t+1) \mathbf{C B}) \delta \mathbf{u}_{k}(t+1) \\
& -\boldsymbol{\Gamma}(t) \mathbf{C A B} \delta \mathbf{u}_{k-1}(t)-\boldsymbol{\Gamma}(t) \sum_{i=0}^{t-1} \mathbf{C A}^{t-i} \mathbf{B} \delta \mathbf{u}_{k}(i) .
\end{aligned}
$$

Expressing $\delta \mathbf{u}_{k+1}(t)$ by means of control error in $(k-1)$ st iteration, we have

$$
\delta \mathbf{u}_{k+1}(t+1)=\left[\begin{array}{c}
\boldsymbol{\Gamma}(t+1) \mathbf{C A}^{t+1} \mathbf{B} \\
\vdots \\
\vdots \\
\Gamma(t+1) \mathbf{C A}^{2} \mathbf{B} \\
\Gamma(t+1) \mathbf{C A B} \\
\mathbf{I}-\boldsymbol{\Gamma}(t+1) \mathbf{C B}
\end{array}\right]^{T}
$$

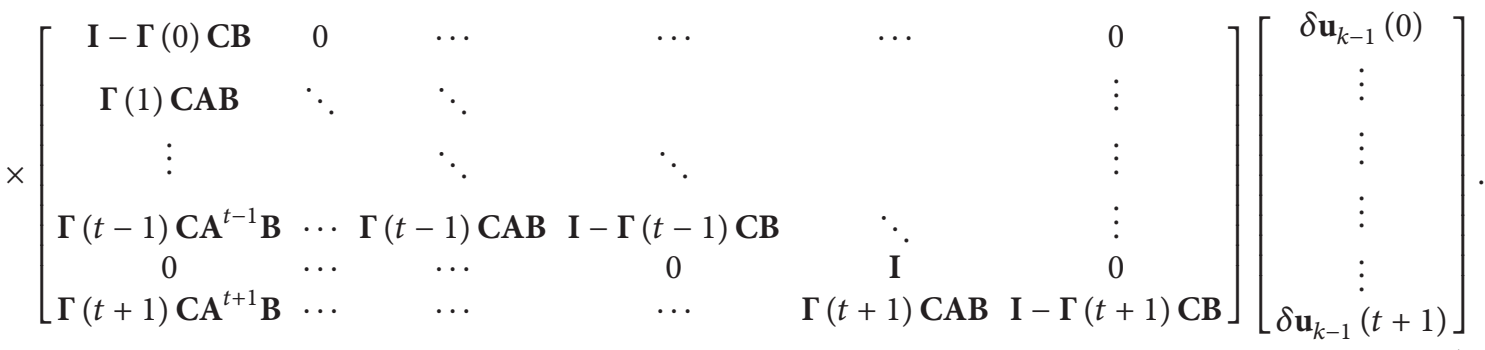

Taking norms of both sides of (31), we have

$$
\begin{aligned}
& \left\|\delta \mathbf{u}_{k+1}(t+1)\right\| \\
& \leq \mathbf{H}_{k+1, k}^{\prime}(t+1) \mathbf{H}_{k, k-1}^{\prime}(t+1) \psi_{k-1}(t+1), \\
& \mathbf{H}_{k+1, k}^{\prime}(t+1) \\
& \quad=\left[\begin{array}{llllll}
\rho_{1}(t+1) & \cdots & \rho_{1}(1) & \left.\rho_{0}(t+1)\right], \\
\mathbf{H}_{k, k-1}^{\prime}(t+1) & & & \\
&
\end{array}\right. \\
& =\left[\begin{array}{cccccc}
\rho_{0}(0) & 0 & \cdots & \cdots & \cdots & 0 \\
\rho_{1}(1) & \ddots & \ddots & & & \vdots \\
\vdots & & \ddots & \ddots & & \vdots \\
\rho_{1}(t-1) & \cdots & \rho_{1}(1) & \rho_{0}(t-1) & \ddots & \vdots \\
0 & \cdots & \cdots & 0 & 1 & 0 \\
\rho_{1}(t+1) & \cdots & \cdots & \cdots & \rho_{1}(1) & \rho_{0}(t+1)
\end{array}\right] .
\end{aligned}
$$

$$
\begin{aligned}
\delta \mathbf{u}_{k+1}(t+1) & (\mathbf{I}-\boldsymbol{\Gamma}(t+1) \mathbf{C B}) \\
& \times\left(\delta \mathbf{u}_{k-1}(t+1)-\boldsymbol{\Gamma}(t+1) \sum_{i=0}^{t+1} \mathbf{C} \mathbf{A}^{t+1-i} \mathbf{B} \delta \mathbf{u}_{k-1}(i)\right) \\
& -\boldsymbol{\Gamma}(t+1) \mathbf{C A B} \delta \mathbf{u}_{k-1}(t)-\boldsymbol{\Gamma}(t+1) \\
& \times \sum_{i=0}^{t-1} \mathbf{C} \mathbf{A}^{t+1-i} \mathbf{B}\left(\delta \mathbf{u}_{k-1}(i)-\boldsymbol{\Gamma}(i) \sum_{j=0}^{i} \mathbf{C A}^{i-j} \mathbf{B} \delta \mathbf{u}_{k-1}(j)\right) .
\end{aligned}
$$

Furthermore, (30) could be rewritten in the form of transition matrix 
By analogy, if multiple control signals are lost at time $t$ in each iteration, which will result in more "1" in the diagonal elements of transition matrix, we also have $\lambda_{m}\left(\prod_{i=0}^{\infty} \mathbf{H}_{i+1, i}(j)\right) \rightarrow 0, m \in[0, j], j \in[t+1, T-1]$, and then $\lim _{k \rightarrow \infty}\left\|\delta \mathbf{u}_{k+1}(i)\right\|=0, i \in[t+1, T-1]$.

Similarly, we can conclude that $\lim _{k \rightarrow \infty}\left\|\delta \widetilde{\mathbf{u}}_{k}(i)\right\|=$ $\lim _{k \rightarrow \infty}\left\|\mathbf{u}_{d}(i)-\mathbf{u}_{k-1}(i)\right\|=0, i \in[t+2, T-1]$ because $\lim _{k \rightarrow \infty} \mathbf{u}_{k}(i)=\lim _{k \rightarrow \infty} \mathbf{u}_{k-1}(i)$. Because the output error $\left\|\mathbf{e}_{k}(t+1+i)\right\|, i \in[1, T-t-1]$ is a function of the control error $\left\|\delta \widetilde{\mathbf{u}}_{k}(j)\right\|, j \in[0, t+i]$, according to (11), the convergence of $\left\|\delta \widetilde{\mathbf{u}}_{k}(j)\right\|, j \in[0, t+i]$, indicates $\lim _{k \rightarrow \infty}\left\|\mathbf{e}_{k}(t+1+i)\right\|$, $i \in[1, T-t-1]$.

Up to now, by compensating the data dropout with the control signal at the same time with the lost one but in the last iteration, the convergence property of output error has been proved. The effect of data dropout was also discussed in [21] by assuming that the actuator is event driven. That is, when there is one signal loss at $t$ during the $k$ th iteration, the signal sent at $t-1$ will continue to be applied in the system before the signal sent at $t+1$ arrives. If $\mathbf{u}_{k}(t)$ is lost, this method can guarantee the control error $\left\|\delta \mathbf{u}_{k}(t)\right\|=0$, $t \in[0, T-1]$, at the controller converge, but the control error $\delta \widetilde{\mathbf{u}}_{k}(t)$ at the actuator cannot converge as iteration goes on because $\lim _{k \rightarrow \infty}\left\|\delta \widetilde{\mathbf{u}}_{k}(t)\right\|=\lim _{k \rightarrow \infty}\left\|\mathbf{u}_{d}(t)-\mathbf{u}_{k}(t-1)\right\| \neq 0$. According to (11), the output error $\left\|\mathbf{e}_{k}(t)\right\|$ cannot converge to zero, and the effect of random data dropouts increases with the increment of random data dropout probability.

\section{Simulation Results}

In this section, some simulation results are provided to illustrate the validity of the proposed method. Let us consider the following discrete-time system:

$$
\begin{aligned}
\mathbf{x}_{k}(t+1)= & {\left[\begin{array}{ccc}
-0.5 & 0 & 0 \\
1 & 1.24 & -0.87 \\
0 & 0.87 & 0
\end{array}\right] \mathbf{x}_{k}(t)+\left[\begin{array}{l}
1 \\
0 \\
0
\end{array}\right] \widetilde{\mathbf{u}}_{k}(t), } \\
& \mathbf{y}_{k}(t)=\left[\begin{array}{lll}
2 & 2.6 & -2.8
\end{array}\right] \mathbf{x}_{k}(t) .
\end{aligned}
$$

The desired trajectory is

$$
\mathbf{y}_{d}(t)=5 \sin \left[\frac{8(t-1)}{T}\right] .
$$

ILC method is described in (5). Initial state error $\delta \mathbf{x}_{k}(0)$ and initial control error $\delta \mathbf{u}_{0}(t)$ are $0, T=200 . \Gamma(t)=0.2$, so $0<\rho(\boldsymbol{\Gamma}(t) \mathbf{C B})=0.4<1$ is satisfied. The mean of output errors $\sum_{t=1}^{T}\left\|\mathbf{e}_{k}(t)\right\|$ in each iteration is selected to show the convergence of output error. In each iteration, $\xi_{k}(t)$ and $\eta_{k}(t)$ take value 0 or 1 according to the data dropout rate correspondingly.

The mean of output errors without any compensation versus iterations is shown in Figure 2. From Figure 2, it is easy to find that the effect of control signal dropouts on the mean of output error increases as random data dropout rate increases. The mean of output errors compensated by the control signal in the same iteration with the lost one but at the last time is shown in Figure 3. The comparison of the
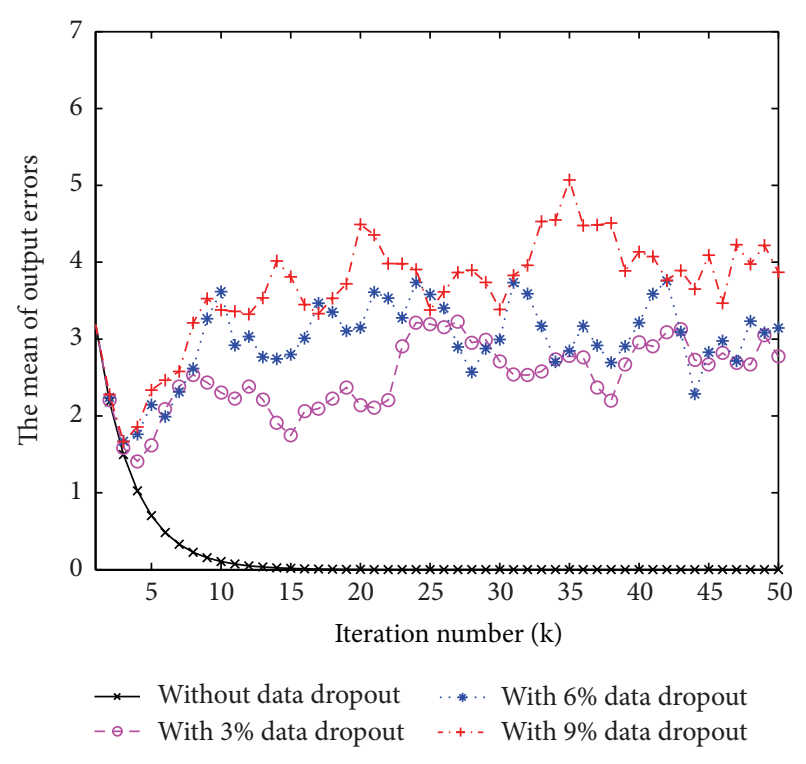

FIGURE 2: Without compensation.

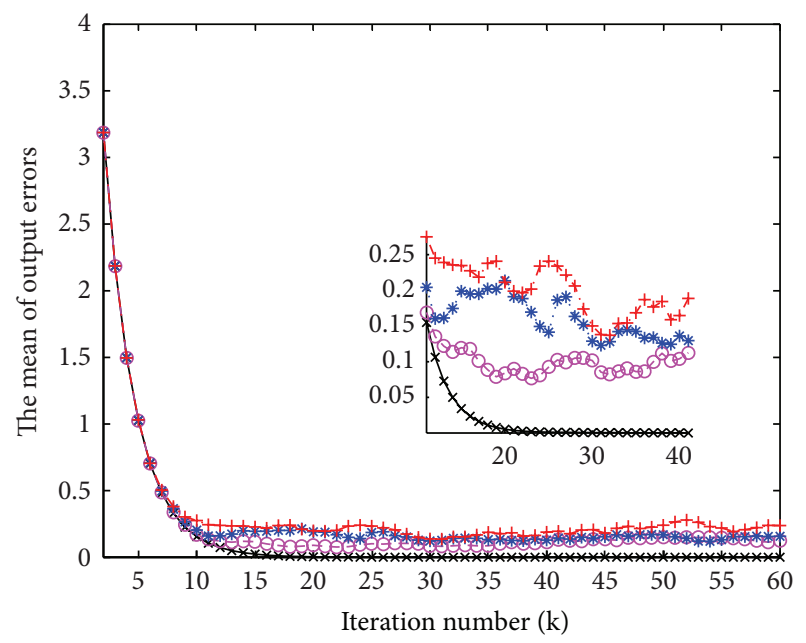

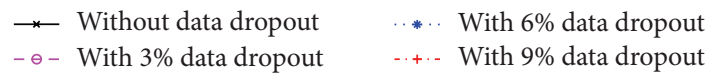

FIGURE 3: Compensated by the signal in the same iteration with the lost one but at the last time.

simulation results in Figures 3 and 2 shows that although the effect of data dropouts is reduced obviously, the mean of output errors does not converge to zero because this method cannot guarantee control error at the actuator converge to zero. And what is more, the effect of data dropouts increases with the increment of dropout rate. The mean of output errors compensated by the control signal at the same time with the lost one but in the last iteration is shown in Figure 4. By comparing Figure 4 with Figures 2 and 3, it is clear that even though the dropout rate would affect the convergence speed of the output error mean, which can still converge to zero from iteration to iteration with different dropout rates. 


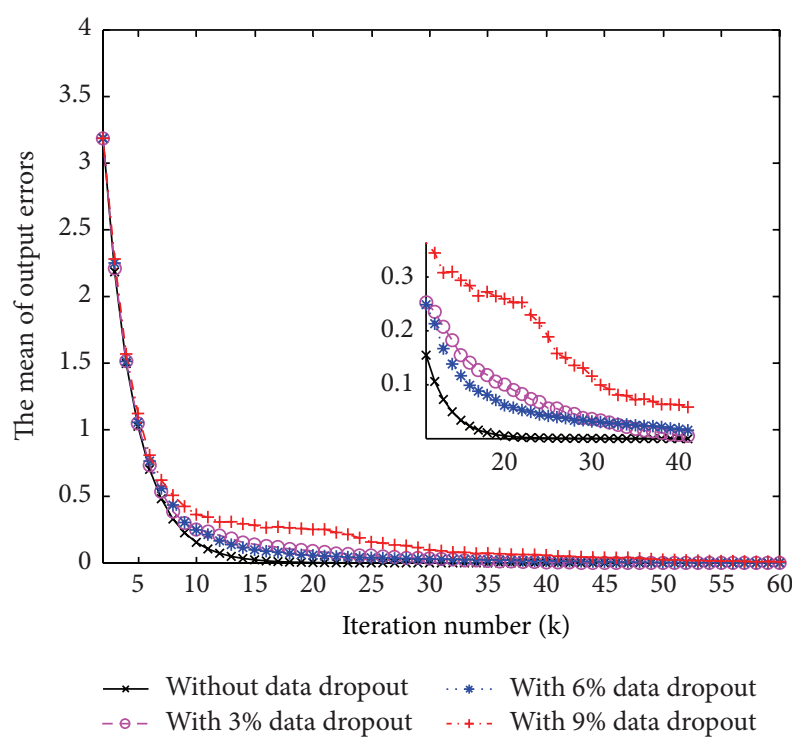

FIGURE 4: Compensated by the signal at the same time with the lost one but in the last iteration.

\section{Conclusions}

In order to remove the effect of data dropouts on the wireless remote ILC systems, a compensation method using the signal at the same time with the lost one but in last iteration is proposed to guarantee the convergence property of output error. The method can not only guarantee the convergence of control error at the controller, but also guarantee the convergence of control error at the actuator. By modeling the random data dropouts as a Bernoulli distributed sequence, the convergence property of control error at the controller is proved through studying the element values of system transition matrix, and then the convergence of control error at the actuator and output error is analyzed. Finally, the method is supported by some simulation results.

In wireless remote ILC systems, channel noises and time delays are other two main issues. In the future, we should consider the effects of data dropouts, channel noises, and time delays simultaneously. Besides, in order to improve the tracking performance further, we may select the learning gain adaptively according to such optimization criterions as least mean square and so on.

\section{References}

[1] Y. Zhang, K. Yang, S. Xie, and H. H. Chen, "Distributed geographical packet forwarding in wireless sensor and actuator networks-a stochastic optimal control approach," IET Wireless Sensor Systems, vol. 2, no. 1, pp. 63-74, 2012.

[2] T. Li and Y. Fujimoto, "Control system with high-speed and realtime communication links," IEEE Transactions on Industrial Electronics, vol. 55, no. 4, pp. 1548-1557, 2008.

[3] S. Oh and S. Sastry, "Distributed networked control system with lossy links: state estimation and stabilizing communication control," in Proceedings of the 45th IEEE Conference on Decision and Control (CDC '06), pp. 1942-1947, San Diego, Calif, USA, December 2006.
[4] S. Arimoto, S. Kawamura, and F. Miyazaki, "Bettering operation of robots by learning," Journal of Robotic Systems, vol. 1, no. 2, pp. 123-140, 1984.

[5] M. Moayedi, Y. K. Foo, and Y. C. Soh, "Adaptive Kalman filtering in networked systems with random sensor delays, multiple packet dropouts and missing measurements," IEEE Transactions on Signal Processing, vol. 58, no. 3, part 2, pp. 1577-1588, 2010.

[6] J. Ma and S. Sun, "Optimal linear estimators for systems with random sensor delays, multiple packet dropouts and uncertain observations," IEEE Transactions on Signal Processing, vol. 59, no. 11, pp. 5181-5192, 2011.

[7] M. Sahebsara, T. W. Chen, and S. L. Shah, "Optimal $H_{2}$ filtering in networked control systems with multiple packet dropout," IEEE Transactions on Automatic Control, vol. 52, no. 8, pp. 1508$1513,2007$.

[8] Y. Liang, T. W. Chen, and Q. Pan, "Optimal linear state estimator with multiple packet dropouts," IEEE Transactions on Automatic Control, vol. 55, no. 6, pp. 1428-1433, 2010.

[9] S. L. Sun, L. H. Xie, W. D. Xiao, and Y. C. Soh, "Optimal linear estimation for systems with multiple packet dropouts," Automatica, vol. 44, no. 5, pp. 1333-1342, 2008.

[10] L. Bakule and M. de la Sen, "Decentralized resilient Ho observer-based control for a class of uncertain interconnected networked systems," in Proceedings of the American Control Conference (ACC '10), pp. 1338-1343, Baltimore, Md, USA, July 2010.

[11] L. Bakule and M. de la Sen, "Decentralized stabilization of networked complex composite systems with nonlinear perturbations," in Proceedings of the IEEE International Conference on Control and Automation (ICCA '09), pp. 2272-2277, Christchurch, New Zealand, December 2009.

[12] W. W. Che and G. H. Yang, "Non-fragile state feedback Ho control with quantized signals via LMI method," in Proceedings of the Chinese Control and Decision Conference (CCDC '08), pp. 3038-3043, Yantai, China, July 2008.

[13] C. P. Liu, J. X. Xu, and J. Wu, "Iterative learning control for remote control systems with communication delay and data dropout," Mathematical Problems in Engineering, vol. 2012, Article ID 705474, 14 pages, 2012.

[14] C. P. Liu, J. X. Xu, and J. Wu, "Iterative learning control for network systems with communication delay or data dropout," in Proceedings of the 48th IEEE Conference on Decision and Control held jointly with 2009 28th Chinese Control Conference (CDC/CCC '09), pp. 4858-4863, Shanghai, China, December 2009.

[15] X. H. Bu and Z. S. Hou, "Stability of iterative learning control with data dropouts via asynchronous dynamical system," International Journal of Automation and Computing, vol. 8, no. 1, pp. 29-36, 2011.

[16] X. H. Bu, Z. S. Hou, and F. S. Yu, "Stability of first and high order iterative learning control with data dropouts," International Journal of Control, Automation, and Systems, vol. 9, no. 5, pp. 843-849, 2011.

[17] H. S. Ahn, Y. Q. Chen, and K. L. Moore, "Intermittent iterative learning control," in Proceedings of the IEEE International Symposium on Intelligent Control (ISIC '06), pp. 832-837, Munich, Germany, October 2006.

[18] H. S. Ahn, Y. Q. Chen, and K. L. Moore, "Discrete-time intermittent iterative learning control with independent data dropouts," in Proceedings of the 17th IFAC World Congress, pp. 12442-12447, Seoul, Korea, July 2008. 
[19] X. H. Bu, F. S. Yu, Z. S. Hou, and F. Z. Wang, "Iterative learning control for a class of nonlinear systems with random packet losses," Nonlinear Analysis: Real World Application, vol. 14, no. 1, pp. 567-580, 2013.

[20] Y. J. Pan, H. J. Marquez, and T. Chen, "Sampled-data iterative learning control for a class of nonlinear networked control systems," in Proceedings of the American Control Conference, pp. 3494-3499, Minneapolis, Minn, USA, June 2006.

[21] Y. J. Pan, H. J. Marquez, T. Chen, and L. Sheng, "Effects of network communications on a class of learning controlled nonlinear systems," International Journal of Systems Science, vol. 40, no. 7, pp. 757-767, 2009.

[22] Y. Fang and H. C. Yan, "Error analysis for remote nonlinear iterative learning control system with wireless channel noise," Journal of Shanghai University, vol. 15, no. 1, pp. 7-11, 2011.

[23] H. C. Yan and F. Yong, "Error estimate for remote ILC system with gauss channel noise," in Proceedings of the 4th International Conference on Intelligent Computation Technology and Automation (ICICTA '11), pp. 540-543, Shenzhen, China, March 2011. 


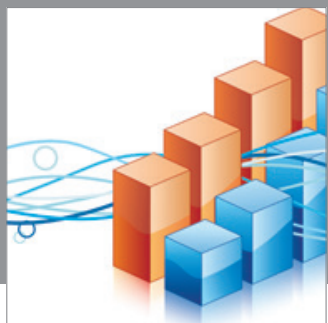

Advances in

Operations Research

mansans

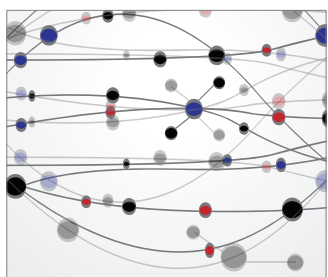

The Scientific World Journal
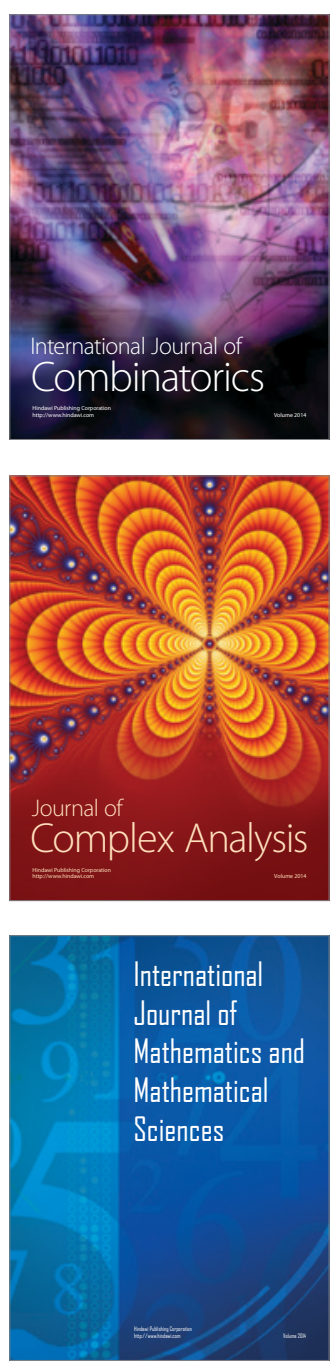
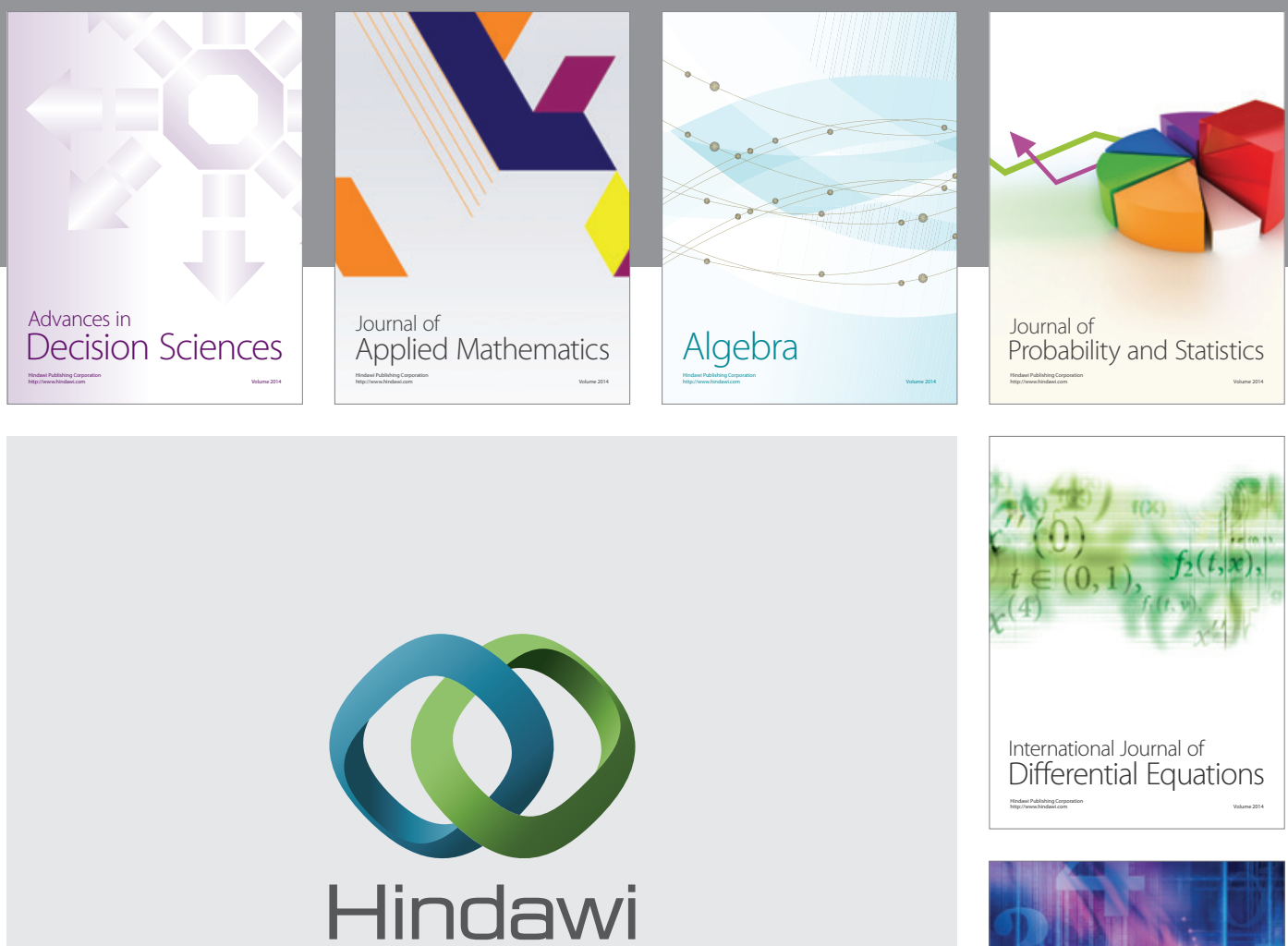

Submit your manuscripts at http://www.hindawi.com
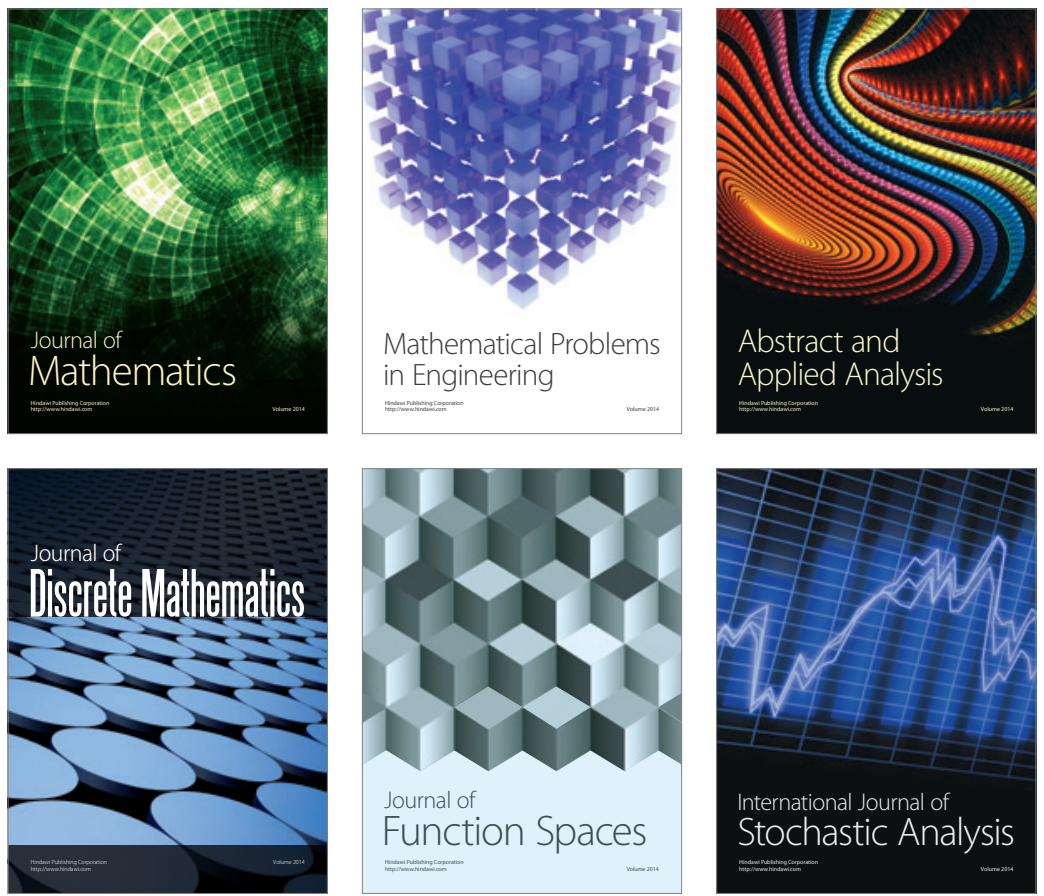

Journal of

Function Spaces

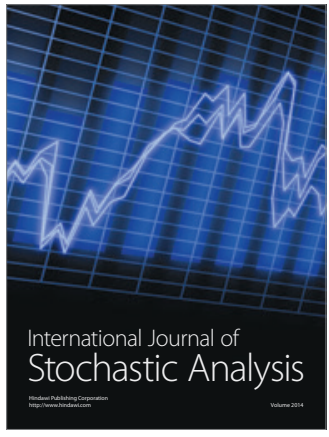

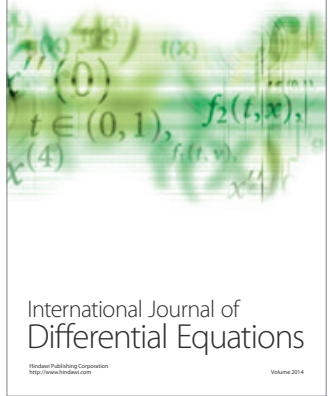
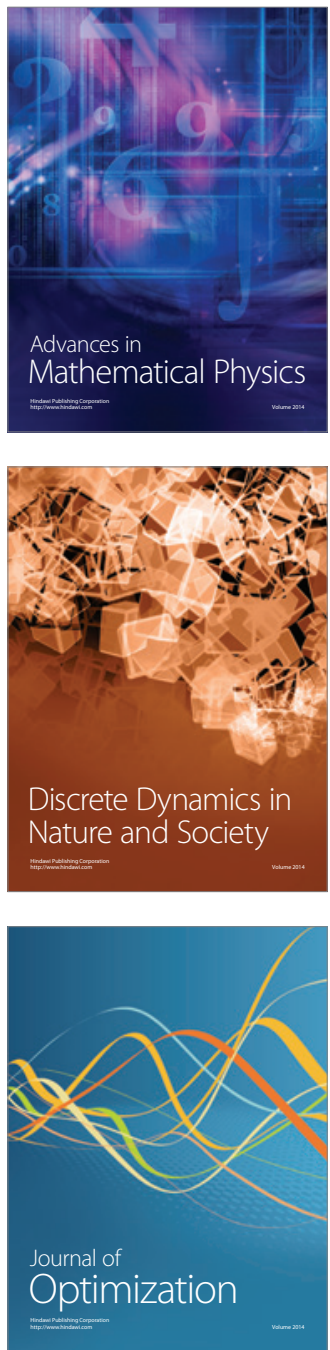